\title{
STRUCTURAL DAMAGE DETECTION IN MOVING LOAD PROBLEM USING JRNNS BASED METHOD
}

\author{
Shakti P. JEnA \\ Department of Mechanical Engineering, Vardhaman College of Engineering, Hyderabad, India \\ e-mail: shaktipjena@gmail.com \\ DAYAL R. PARHI \\ Department of Mechanical Engineering, National Institute of Technology, Rourkela, India \\ e-mail:dayalparhi@gmail.com
}

\begin{abstract}
Damage detection in a structure using the vibration signature is a quiet smart method for condition monitoring of the structure. In this problem, the Recurrent Neural Networks (RNNs) based method has been implemented for damage detection in the moving load problem as an inverse method. A multi-cracked simply supported beam under a traversing load has been considered for the present problem. The localization and severities of the supervised cracks on the structure are determined using the adapted Jordan's Recurrent Neural Networks (JRNNs) approach. The mechanism of Levenberg-Marquardt's back propagation algorithm has been implemented to train the networks. To check the adoptability of the proposed JRNNs method, numerical analyses along with laboratory test verifications have been conducted and found to be well emerged.
\end{abstract}

Keywords: Jordan's Recurrent Neural Networks (JRNNs), crack depth, crack location

\section{Introduction}

Cracks are induced during the service period and mechanized progression of structures. So, the early detection of a crack is essential in order to sustain the stability and dynamic behavior of structures. In the current time, applications of artificial neural networks have been widely used for fault analysis in dynamic structure identification problems. So fault detection by using a vibration signature is extremely smart for supervising bridge structures because it presents the prospect of electronic recording along with digital processing and information creation. Many researchers have been working in the area of crack detection using various techniques of the forward as well as reverse manner.

Krawczuk and Ostachowicz (1995) developed a discrete and finite element model for a composite structure with the presence of an edge crack. They discussed various factors like depth of the crack, volume fraction of fibers and orientation of angles in the composite structure and their significance on the response of the beam. Dems and Mróz (2001) devoted their work on damage identification procedures by implementing the mechanism of factors associated with the natural frequency of the structure. Owolabi et al. (2003) formulated a damage detection technique in structures using changes in natural frequencies and amplitudes of frequency response functions. Seker et al. (2003) have studied the ability of Elman's recurrent neural network to diagnose faults and conditions of a nuclear power plant and rotating machine structures. Malhi and Gao (2004) approached recurrent neural networks to predict the condition by monitoring of machines. Using the modal properties, a neural network method was proposed for damage identification in a bridge structure by Lee et al. (2005) by considering errors occurring in baseline finite element models. 
Utilizing the concepts of neural networks for pattern recognition of the vibration signature, Yeung and Smith (2005) developed a damage detection procedure for bridge-like structures. They simulated them with a real damage scenario and evaluated the responses of the structure under moving traffic conditions. Bu et al. (2006) proposed an inventive method to calculate the bridge state from the response of a traversing vehicle. Yan et al. (2007) presented different types of structural damage detection methods based on using vibration signatures. Mehrjoo et al. (2008) presented a method to determine damage intensities of joints in a truss bridge structure using an artificial neural network based on the back propagation rule.

$\mathrm{Hu}$ and Balasubramaniam (2008) analyzed some real case studies on the application of Elman's recurrent neural network. Ekici et al. (2009) determined the fault location happening in a transmission line using Elman's recurrent network (ERN). Prakrashi et al. (2010) investigated a damage evolution method by experimental monitoring of vehicle bridge interaction dynamics.

Sayyad and Kumar (2011) carried out analytical and experimental methods for crack detection in a simple supported beam with a single crack by analyzing the concepts of measurement of natural frequencies. They used this method as an inverse problem to determine the crack locations and size for different types of the structure.

González-Pérez and Valdés-González (2011) presented a structural damage identification procedure in a bridge structure under vehicular load using the artificial neural network method. Li and Law (2012) presented a substructure crack detection method from the dynamic response reconstruction technique of a passing vehicle. Li et al. (2013) conducted a numerical study along with experimental investigation for fault diagnosis in a bridge structure under a moving load using the dynamic reconstruction method in the wavelet domain. Coban (2013) proposed a novel context layer locally recurrent neural network (CLLRNN) method for the dynamic system identification problem using the back propagation algorithm with an adaptive learning rate.

Bandara et al. (2014) developed an artificial neural network based technique for damage identification in a structure using the frequency response function. Oshima et al. (2014) developed an indirect approach to study the state of a bridge based on the mode shape analysis by the responses of travelling vehicular loads. Nandakumar and Shankar (2014) presented a damage detection system for a double cracked beam using the two crack transfer matrix method. Ettefagh et al. (2014) investigated a crack detection procedure for structures based on modal updating and the model reduction method.

Lee and $\mathrm{Wu}$ (2014) found out natural frequencies of a cracked beam using the local adaptive differential quadrature method. In the later part, they employed Newton-Raphson's iteration method for fault isolation in multiple cracked structures. Kong et al. (2015) carried out a computational study to explore the possibilities of damage in a bridge by the vehicle transmissibility of the vehicle bridge couple system. Vosoughi (2015) investigated a novel technique to identify cracks in beams by hybridizing genetic algorithms (GAs) and particle swarm optimization (PSO) methods. He and Zhu (2016) explored a closed form solution to analyze the dynamic response of a cracked simple supported beam subjected to a moving load. Further, they extended this problem for damage detection in a beam based on the response of the induced moving load.

Jena and Parhi (2017a,b,c), Jena et al. (2015, 2017), Parhi and Jena (2017) carried out theoretical analyses along with the FEA and experimental verifications methods to determine responses of a damaged structure with different end conditions under transit loading conditions. They also investigated the consequences of different parameters (moving speed, magnitude of the moving mass, crack positions and crack severities) and their influences on the responses of the structure. Mousavi and Gandomi (2018) developed a novel damage detection procedure by directly using the input and output parameters of the forced vibrating structure.

As far the literature is concerned, to the best of the author's knowledge, damage detection in structural dynamics problems using the concepts of RNNs is little. The proposed analogy applies the concepts of Jordan's Recurrent Neural Networks (JRNNs) with the L-M back propagation 
algorithm as a reverse problem to detect and localize some damage parts in the structure under a moving mass. The present crack detection approach has been carried out in a supervised mode by considering a simply supported structure under the transiting mass. The severities of the cracks are also determined.

\section{Problem formulation}

In the present investigation, a simply supported beam (Fig. 1) with multiple cracks subjected to a traversing load has been considered for the analysis. The responses of the simply supported beam subjected to the traversing load are found out both theoretically and experimentally. The theoretical problem has been solved by approaching the concepts of Euler-Bernoulli's assumptions.

(a)

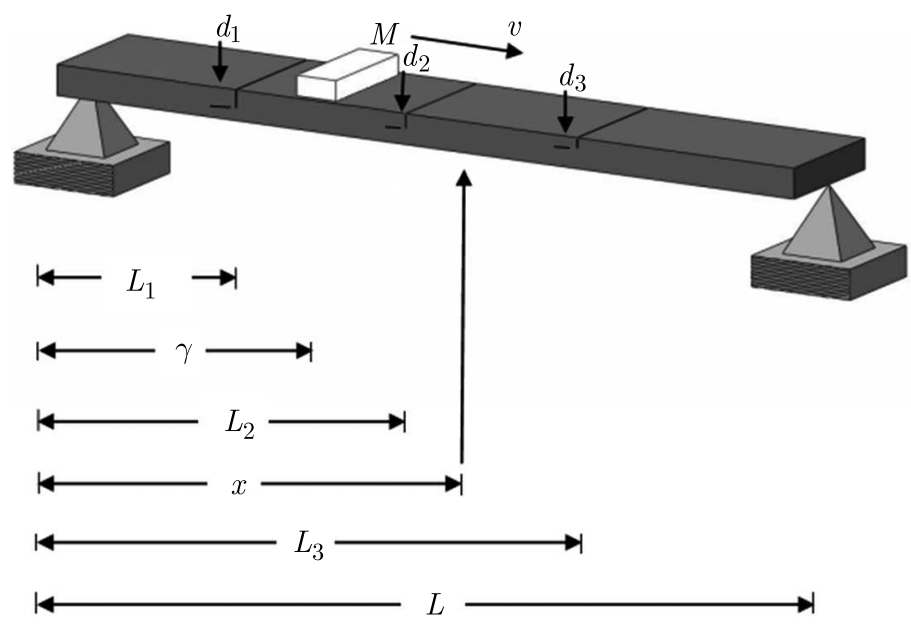

(b)

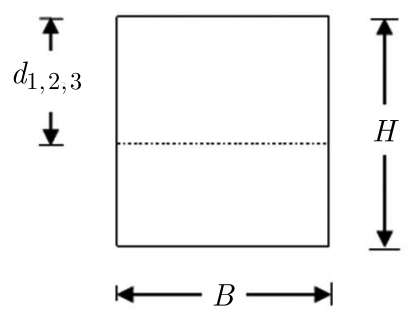

Fig. 1. (a) Schematic presentation of a cracked simply supported beam under a traversing mass.

(b) Schematic presentation of the cross-section of the cracked simply supported beam

The equation of motion of a structure subjected to a traversing load is given by

$$
E I \frac{\partial^{4} y}{\partial x^{4}}+m \frac{\partial^{2} y}{\partial t^{2}}=F(x, t)
$$

where $F(x, t)=P(t) \delta(x-\gamma)+r(x, t)$ - interactive force due to the traversing mass, and $r(x, t)-$ universal loading conditions (zero in the present investigation), $\delta$ - Dirac delta function, $\gamma=v t$ - position of the traversing mass at any moment of time $t . P(t)$ is the applied force due to the moving mass

$$
P(t)=M g-M\left(\frac{\partial}{\partial t}+v \frac{\partial}{\partial \gamma}\right)^{2} y(\gamma, t)
$$

where $\partial^{2} y(\gamma, t) / \partial t^{2}$ - inertial acceleration, $v^{2} \partial^{2} y(\gamma, t) / \partial \gamma^{2}$ - centrifugal acceleration, $2 v \partial^{2} y(\gamma, t) /(\partial \gamma \partial t)$ - Coriolis acceleration, $H$ - thickness of the beam, $B$ - width of the beam, 
$M$ - traversing mass, $v$ - traversing speed, $m$ - beam mass per unit length, $g$ - gravitational constant, $L$ - length of the beam, $L_{1}, L_{2}, L_{3}$ - locations of the first, second and third crack from the left supported end of the cracked simply supported structure, respectively, $d_{1}, d_{2}, d_{3}$ - depth of the first, second and third crack, respectively.

The general elucidation of equation (2.1) has been written as in series from, i.e.

$$
y(x, t)=\sum_{n=1}^{\infty} \varphi_{n}(x) T_{n}(t)
$$

where $\varphi_{n}(x)$ - eigen function of the structure without allowing the moving mass, $T_{n}(t)$ - function of amplitude to be determined, $n$ - number modes of vibrations.

For the evaluation of $\varphi(x)$, equation (2.2) has been expressed as

$$
\varphi_{n}^{i v}(x)-\lambda_{n}^{4} \varphi_{n}(x)=0
$$

where

$$
\lambda_{n}^{4}=\rho A \frac{\omega_{n}^{2}}{E I}=m \frac{\omega_{n}^{2}}{E I}
$$

After some rearrangements and simplifications, the final solution to equation (2.2) has been expressed below

$$
E I \lambda_{n}^{4} T_{n}(t)+m T_{n, t t}(t)-\left(\frac{M}{S_{n}}\right)\left[g-\sum_{q=1}^{\infty}\left(\frac{\partial}{\partial t}+v \frac{\partial}{\partial \gamma}\right)^{2} \varphi_{q} T_{q}(t)\right] \varphi_{n}(\gamma)=0
$$

Equation (2.4), which is a fourth order differential equation, has been earlier solved by Jena and Parhi (2017b) by using the fourth order Runge-Kutta method. By solving equation (2.4), the responses of the structure are determined. To corroborate the proposed computational approach, an experiment has also been conducted in the laboratory.

The laboratory test model is shown in Fig. 2. The same laboratory test procedures are carried out here as those of Jena and Parhi (2017b) who previously found out the responses of the structure. For the above theoretical and experimental works, numerical examples are formulated to predict the subsistence and positions of cracks in the structure as in the forward manner.

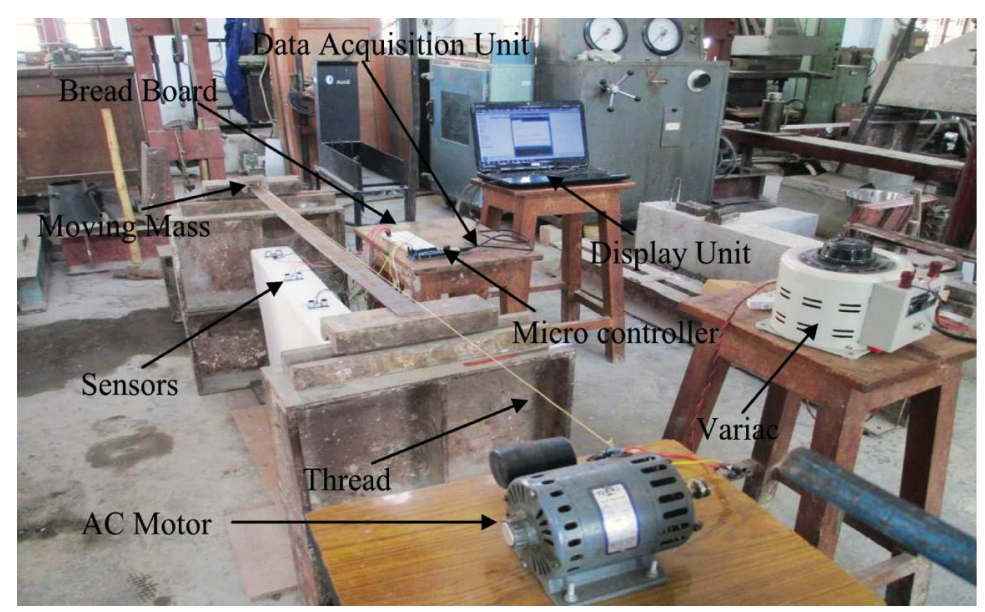

Fig. 2. Laboratory test model for a simply supported structure 


\section{Numerical formulation}

- Beam type - rectangular (mild steel specimen)

- Beam size $-140 \mathrm{~cm} \times 4.5 \mathrm{~cm} \times 0.5 \mathrm{~cm}$

- Moving speed $-4.18 \mathrm{~m} / \mathrm{s}$ and $5.13 \mathrm{~m} / \mathrm{s}$

- Moving mass - $1.9 \mathrm{~kg}$ and $1.15 \mathrm{~kg}$

- Crack locations $\left\{L_{1}, L_{2}, L_{3}\right\}=\{51,72.1,89.2\} \mathrm{cm}$ and $\left\{L_{1}, L_{2}, L_{3}\right\}=\{63.7,84.9,106.1\} \mathrm{cm}$

- Relative crack depth $\left\{\alpha_{1}, \alpha_{2}, \alpha_{3}\right\}=\{0.18,0.32,0.43\}$ and $\left\{\alpha_{1}, \alpha_{2}, \alpha_{3}\right\}=\{0.26,0.45,0.54\}$, where $\alpha_{i}=d_{i} / h(i=1,2,3)$

The forward problem analyses are done both theoretically and experimentally. The dynamic responses of the cracked beam structure due to the movement of the traversing mass are also determined. The deflections of the structure with respect to the positions of the traversing mass are determined and shown in Figs. 3a and 3b. From the illustrations in Figs. 3a and 3b, there has been observed that, to some extent, there are sudden intensifications in the dynamic deflections or amplitudes.

(a)

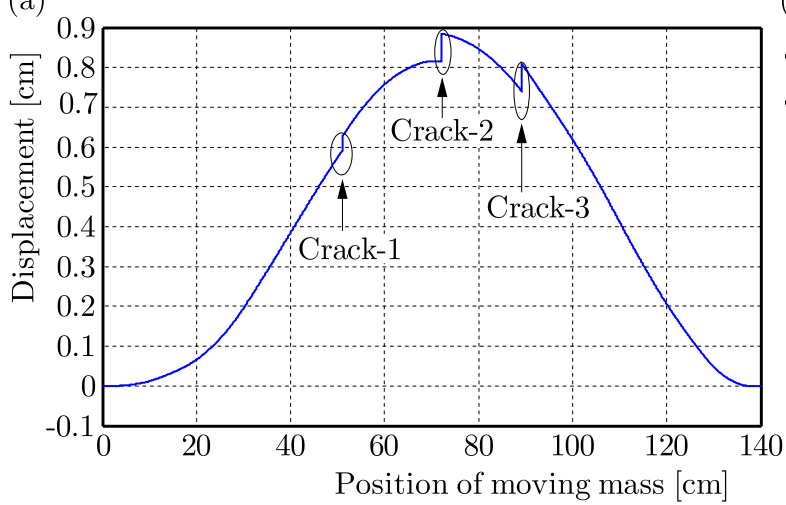

(b)

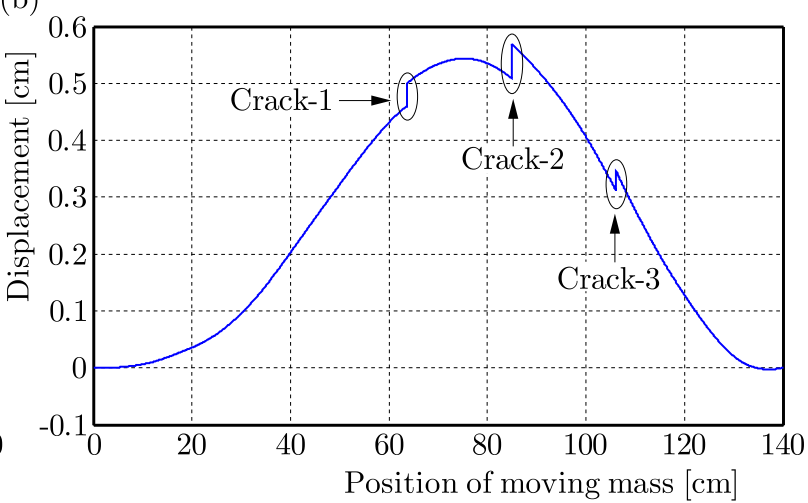

Fig. 3. Displacement vs. position of the moving mass for $M=1.9 \mathrm{~kg}$ : (a) $v=4.18 \mathrm{~m} / \mathrm{s}$, $\left\{\alpha_{1}, \alpha_{2}, \alpha_{3}\right\}=\{0.18,0.32,0.43\},\left\{L_{1}, L_{2}, L_{3}\right\}=\{51,72.1,89.2\} ;$ (b) $v=5.13 \mathrm{~m} / \mathrm{s}$, $\left\{\alpha_{1}, \alpha_{2}, \alpha_{3}\right\}=\{0.26,0.45,0.54\},\left\{L_{1}, L_{2}, L_{3}\right\}=\{63.7,84.9,106.1\}$

From this kind of behavior in the beam deflections, it is proved that there are some possible existences of cracks on the structure. The positions of the cracks are found out from the deliberated dynamic responses of the structures, i.e. sudden amplifications in dynamic deflections to the corresponding positions of the traversing mass. The severities of the cracks are observed by the experimental study.

\section{Application of Jordan's Recurrent Neural Networks (JRNNs) for crack detection in the structure}

In the present problem, knowledge based JRNNs have been proposed for crack detection in the moving-load dynamic problem. The key advantage of networks are the feedback links which use information later. The adapted architecture of the proposed JRNNs model is shown in Fig. 4. In JRNNs types of the network structure, there is a feedback connection from the output layer to the hidden layer which is known as the context layer. Due to the adaption of the context layer, the information can be gathered and reused later. In the present analogy, the architectural structure of the JRNNs model is slightly modified. The present JRRNs model has one input and output, two context layers and three hidden layers. Apart from the input and hidden layers, 
self recurrent connections are also provided in the context and output units. Due to the self recurrence and feedback links, dynamic memory has been introduced to the networks unit which behaves as additional memory units. The feedback and self recurrent links have one time delay unit $\left(Z^{-1}\right)$. The numbers of neurons in the context and output layers are the same because the exact information can be copied due to the feedback links. The neurons from the output layer provide information to the context layer-1 through feedback connections and forward these output values to the first hidden layer. Again the context layer-1 supplies information to the context layer-2 and again the output of the context layer- 2 is feed forwarded to the first hidden layer. So, due to the feedback links (context layers 1 and 2) and self recurrence, dynamic memory is provided to the network structure. The Levenberg-Marquardt (Yu and Wilamowski, 2011) back propagation algorithm is implemented here to train the proposed JRNNs structure. Throughout the training procedures of the proposed network structure, the training patterns are fed forward the following components to encompass the networks, and the network is operated accordingly. In Fig. 4, the architectural model of the proposed JRNNs is shown. The layer-1 (input layer) of the proposed model contains the responses of the structure (relative deflection) along with different moving parameters at various damage and undamaged conditions of the structure.

The training parameters are described as follows:

- $t$ - total travelling time of the traversing mass to cross the beam

- RD (Relative Deflection) - deflection of the cracked beam to the uncracked beam at a particular instant of time with the same moving speed and mass

- RD- 1 - relative deflections of the beam at time $t / 8, \mathrm{RD}-2$ - at time $t / 4, \mathrm{RD}-3$ - at time $3 t / 8$, RD-4 - at time $t / 2, \mathrm{RD}-5$ - at time $5 t / 8, \mathrm{RD}-6$ - at time $3 t / 4$

- $W_{1}=\mathrm{RD}-1, W_{2}=\mathrm{RD}-2, W_{3}=\mathrm{RD}-3, W_{4}=\mathrm{RD}-4, W_{5}=\mathrm{RD}-5, W_{6}=\mathrm{RD}-6$

- $W_{7}$ - length of the beam $L$

- $W_{8}$ - speed of the moving mass $v$

- $W_{9}$ - magnitude of the moving mass $M$

- $\varphi_{1}$ - first crack location $L_{1}, \varphi_{2}$ - relative first crack depth $\alpha_{1}$

- $\varphi_{3}$ - second crack location $L_{2}, \varphi_{4}$ - relative second crack depth $\alpha_{2}$

- $\varphi_{5}$ - third crack location $L_{3}, \varphi_{6}$ - relative third crack depth $\alpha_{3}$

- $i=1,2, \ldots, N, N$ is the total number of input nodes

- $r_{1}=r_{2}=1,2, \ldots, R, R$ is the total number of nodes in the context layer-1 and layer-2 (constant)

- $k=1,2, \ldots, O, O$ is the total number of nodes in the output

- $j_{1}=j_{2}=j_{3}=1,2, \ldots, S, S$ is the total number of neurons or nodes in each hidden layer, i.e. the first, second and third hidden layers (constant for all the nodes)

- $\beta$ is the self-recurrent value for each node in the output and context layers that varies from 0 to 1

- $Z^{-1}$ is the unit delay

- $U_{1-6}$ - values of the context units in the context layer

- $\varphi_{r 1}^{t}$ and $\varphi_{r 1}^{t-1}$ are the output values of the nodes of the context layer-1 at the time index $t$ and $t-1$, respectively

- $\varphi_{r 2}^{t}$ and $\varphi_{r 2}^{t-1}$ are the output values of the nodes of the context layer-2 at the time index $t$ and $t-1$, respectively

- $\varphi_{j}^{t}$ is the output values of the hidden node $j$ at the time index $t$

- $\varphi_{k}^{t}$ and $\varphi_{k}^{t-1}$ are the output values of the nodes of the output layer at the time index $t$ and $t-1$, respectively

- $t-1$ is the time index that is deferred by one-time step due to the feedback connections, which is represented by $Z^{-1}$ in the network architecture

- $w$ is the weight of connection. 


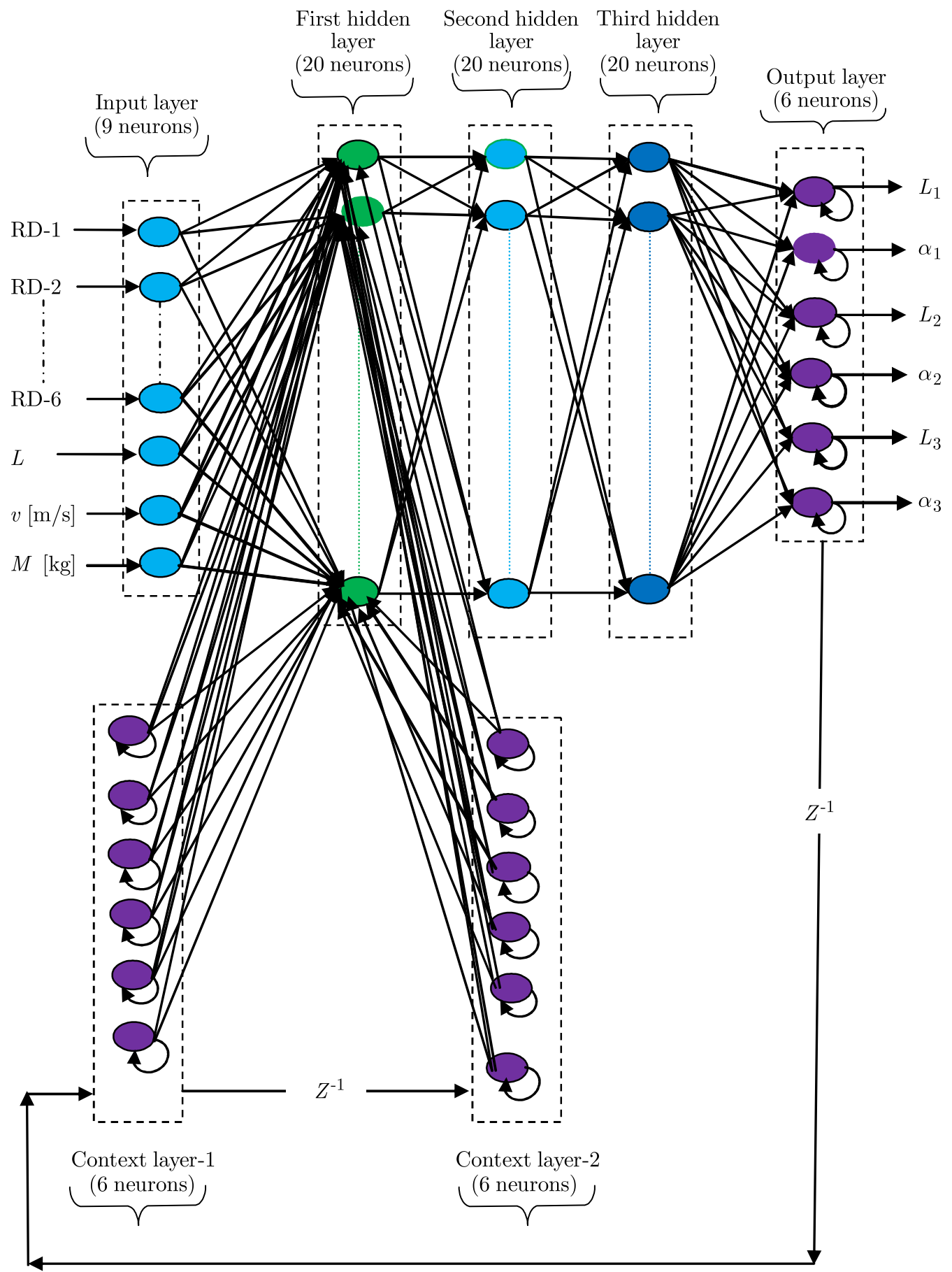

Fig. 4. Adapted architecture of the JRNNs model

By analyzing the network structure (Fig. 4), the mathematical equations for the proposed structure are developed as follows

$$
\varphi_{r 1}^{t}=\varphi_{k}^{t-1}+\beta \varphi_{r 1}^{t-1} \quad \varphi_{r 2}^{t}=\varphi_{r 1}^{t-1}+\beta \varphi_{r 2}^{t-1}
$$

The net input to the first hidden layer of the network model is given by

$$
\varphi_{j 1}^{t}=\sum_{i=1}^{N} W_{i}^{t} w_{i, j 1}+\sum_{r=1}^{R} \varphi_{r 1}^{t} w_{r 1, j 1}+\sum_{r=1}^{R} \varphi_{r 2}^{t} w_{r 2, j 1}
$$


The net input to the first hidden layer of the network model is given by

$$
\varphi_{j 2}^{t}=\sum_{j 1=1}^{S} \varphi_{j 1}^{t} w_{j 1, j 2}
$$

The net input to the third hidden layer or the network model is given by

$$
\varphi_{j 3}^{t}=\varphi_{j}^{t}=\operatorname{net}_{j}=\sum_{j 2=1}^{S} \varphi_{2}^{t} w_{j 2, j 3} \quad \varphi_{j}^{t}=f\left(\text { net }_{j}^{t}\right)
$$

The net output of the network model is given by

$$
\operatorname{net}_{k}^{t}=\sum_{j 3=1}^{S} \varphi_{j 3}^{t} w_{j 3, k}+\beta \varphi_{k}^{t-1} \quad \varphi_{k}^{t}=g\left(\text { net }_{k}^{t}\right)
$$

The activation functions symbols used in the hidden and output layers are $f(\cdot)$ and $g(\cdot)$, respectively. The activation function implemented for the hidden and context layers is "tan-sigmoid" while that in the output layer is "purelin" function. The approximate error function $\varepsilon$ of the output nodes is optimized by implementing the efficient weight factors relation, i.e., $w^{\text {new }}=w^{\text {old }}+\zeta \Delta w$, where $\zeta$ is the learning rate which is a variable and lies between 0 to 1 . The sum square error function is exemplified to assess the training procedures. The Levenberg-Marquardt (L-M) back propagation algorithm ( $\mathrm{Yu}$ and Wilamowski, 2011) is implemented to the proposed network to estimate the crack locations and depth. The L-M back propagation algorithm is fast and having steady convergence. This algorithm coalesces the minimization of two algorithms (steepest descent and Gauss-Newton methods). This approach adopts the rapidity of perfection of the Gauss-Newton method and stability of the steepest descent method. This algorithm accomplishes an interactive training progression between the two algorithms (steepest descent and Gauss-Newton methods).

The equation of the L-M back propagation mechanism (Yu and Wilamowski, 2011) is given by

$$
w_{k+1}=w_{k}-\left(J_{k}^{\mathrm{T}} J_{k}+\xi I\right)^{-1} J_{k} e_{k}
$$

where $\mathbf{J}$ is the Jacobian matrix which has been determined from the Gauss-Newton approach. $\mathbf{I}$ is the identity matrix. $\xi$ is the combination coefficient that if the value of $\xi$ estimates to zero, then equation (4.6) will perform like the method of Gauss-Newton. If $\xi$ is quite large, then it will work as the steepest descent method. $\xi=1 / \nu, \nu$ is the step size or training constant, e - error vector

$$
\mathbf{e}=\varphi_{\text {desired }}-\varphi_{\text {actual }}
$$

where $\boldsymbol{\varphi}_{\text {desired }}$ is the required output vector, $\boldsymbol{\varphi}_{\text {actual }}$ is the actual output vector

$$
\varepsilon=\text { error function }=\frac{1}{2} \sum_{\substack{\text { all training } \\ \text { patterns }}} \sum_{\substack{\text { all } \\ \text { outputs }}} \mathrm{e}^{2}
$$

The training procedures are carried out using the sum square error function. As per the error function rule, if the error value is less than the previous error, the quadratic estimation on the sum error is executing, and the value of $\xi$ should be decreased to minimize the significance of the gradient descent section. But, if the error value is greater than the previous error, then it is required to enhance the value of $\xi$. 
To train the proposed network with the adopted algorithm, 750 numbers of patterns are generated in this analogy for the training process. The patterns are generated by considering different parameters for the moving load dynamic problem. Out of 750 patterns, 650 patterns are selected for training procedures while the remaining 100 patterns are for the testing process. The examples of some patterns are represented in Table 1. The patterns are generated for cracked as well as uncracked beams under the moving mass. The generations of patterns are also aimed at for the beam having single and multiple cracks. During the training process, the numbers of nodes in the input, each for the context, hidden and output layers are 9, 6, 20 and 6, respectively. The selection of the neurons in each hidden layer are favored by the iteration process during the training of the network and found to be 20 as suitable. Implementing the proposed L-M algorithm, the entire training procedures are carried out.

Table 1. Training patterns for the JRNNs structure

\begin{tabular}{|c|c|c|c|c|c|c|c|c|c|c|c|c|c|c|}
\hline \multicolumn{1}{|c|}{ Input parameters } & \multicolumn{5}{|c|}{ Output parameters } \\
\hline RD-1 & RD-2 & RD-3 & RD-4 & RD-5 & RD-6 & $L$ & $v$ & $M$ & $L_{1}$ & $L_{2}$ & $L_{3}$ & $\alpha_{1}$ & $\alpha_{2}$ & $\alpha_{3}$ \\
\hline \hline 1 & 1.16 & 1.09 & 1.1 & 1.13 & 1.17 & 190 & 5.8 & 1.5 & 25 & 45 & 60 & 0.15 & 0.3 & 0.45 \\
\hline 1 & 1 & 1.09 & 1.24 & 1.51 & 1.82 & 160 & 5 & 2.5 & 50 & 65 & 90 & 0.25 & 0.35 & 0.45 \\
\hline 1 & 1.15 & 1.32 & 1.26 & 1.25 & 1.51 & 130 & 6.3 & 2.3 & 40 & 50 & 60 & 0.4 & 0.4 & 0.4 \\
\hline 1 & 1 & 1 & 1.12 & 1.13 & 1.44 & 180 & 5.5 & 3.5 & 77 & 105 & 135 & 0.35 & 0.42 & 0.27 \\
\hline 1 & 1 & 1 & 1.16 & 1.42 & 1.68 & 120 & 5.7 & 2.2 & 50 & 70 & 0 & 0.43 & 0.37 & 0 \\
\hline 1 & 1 & 1 & 1 & 1.15 & 1.27 & 170 & 4.9 & 2.3 & 85 & 0 & 0 & 0.34 & 0 & 0 \\
\hline 1 & 1 & 1 & 1 & 1 & 1 & 190 & 6 & 4 & 0 & 0 & 0 & 0 & 0 & 0 \\
\hline
\end{tabular}

\section{Results and discussions}

A noble fault detection method using the concepts of RNNs approach has been carried out in the present study. The formulation of the present problem has been done in a supervised manner. To corroborate the above said technology (JRNNs), a small problem has been exemplified. The numerical problem which has been considered for theoretical and experimental procedures, has also been considered for the validity of the proposed JRNNs method. Detailed numerical and experimental analyses of the present investigation have been elaborated in the problem formulation part. The existence, location and quantifications of cracks have also been carried out as in the forward problem. The existence and locations of cracks have been obtained from the measured dynamic responses of the structure (Figs. 3a and 3b).

A modified architecture of the JRNNs with the implementation of L-M back propagation has been designed for this investigation. The equations have also been developed for the proposed model. 750 numbers of patterns have been introduced for the training and testing procedures of the JRNNs model. The patterns have been generated for structures having null, single and multiple cracks. The detailed of the patterns are explained in Table 1. In Table 1, the output parameters $L_{1}=L_{2}=L_{3}=0$ and $\alpha_{1}=\alpha_{2}=\alpha_{3}=0$ symbolize that there is no crack in the structure. Similarly, $L_{3}=\alpha_{3}=0$ symbolize the presence of single cracks, $L_{2}=L_{3}=0$ and $\alpha_{2}=\alpha_{3}=0$ indicate the presence of double cracks, and the rest of these triple cracks. The problem is designed to detect and localize some damage parts of the structure up to the presence of three cracks. Appropriate cares are taken during the training procedures of the networks. The sum square error method has been employed to check the error values of the output. 1000 numbers of iterations have been carried out for this problem. The results obtained from each analysis (experimental and JRNNs) have been compared with the numerical results and represented in Tables 2 and 3. For crack locations, the results obtained from JRNNs and experiments 
differ in errors nearly by about $1.1 \%$ and $0.5 \%$, respectively, with those of numerical simulation. For relative crack depth these are about 4.85 and 2.27 , respectively, which are seemed to be convergent. The graphs for the number of iterations and percentage error values are represented in Fig. 5a (crack locations) and Fig. 5b (relative crack depth). This proposed technique can identify the possible locations of cracks and find out severities of cracks to the structure subjected to a transiting mass.

Table 2. Comparison of the results for different crack locations

\begin{tabular}{|c|c|c|c|c|c|c|c|c|}
\hline \multicolumn{3}{|c|}{ Numerical } & \multicolumn{3}{c|}{ Experimental } & \multicolumn{3}{c|}{ JRNNs } \\
\hline$L_{1}$ & $L_{2}$ & $L_{3}$ & $L_{1}$ & $L_{2}$ & $L_{3}$ & $L_{1}$ & $L_{2}$ & $L_{3}$ \\
\hline \hline 51 & 72.1 & 89.2 & 50.73 & 71.83 & 88.74 & 50.41 & 71.34 & 88.52 \\
\hline 63.7 & 84.9 & 106.1 & 63.29 & 84.62 & 105.81 & 62.94 & 84.17 & 105.013 \\
\hline \multicolumn{2}{|c|}{ Average percentage of error } & $0.64 \%$ & $0.36 \%$ & $0.41 \%$ & $1.16 \%$ & $1.04 \%$ & $1.03 \%$ \\
\hline \multicolumn{3}{|c|}{ Total percentage of error } & \multicolumn{3}{c|}{$0.47 \%$} & \multicolumn{3}{c|}{$1.076 \%$} \\
\hline
\end{tabular}

Table 3. Comparison of the results for various relative crack depth

\begin{tabular}{|c|c|c|c|c|c|c|c|c|}
\hline \multicolumn{3}{|c|}{ Numerical } & \multicolumn{3}{c|}{ Experimental } & \multicolumn{3}{c|}{ JRNNs } \\
\hline$\alpha_{1}$ & $\alpha_{2}$ & $\alpha_{3}$ & $\alpha_{1}$ & $\alpha_{2}$ & $\alpha_{3}$ & $\alpha_{1}$ & $\alpha_{2}$ & $\alpha_{3}$ \\
\hline \hline 0.18 & 0.32 & 0.43 & 0.176 & 0.313 & 0.421 & 0.171 & 0.303 & 0.411 \\
\hline 0.26 & 0.45 & 0.54 & 0.253 & 0.439 & 0.529 & 0.248 & 0.427 & 0.515 \\
\hline \multicolumn{2}{|c|}{ Average percentage of error } & $2.44 \%$ & $2.31 \%$ & $2.065 \%$ & $4.81 \%$ & $5.21 \%$ & $4.524 \%$ \\
\hline \multicolumn{3}{|c|}{ Total percentage of error } & \multicolumn{3}{|c|}{$2.261 \%$} & \multicolumn{3}{c|}{$4.846 \%$} \\
\hline
\end{tabular}

(a)

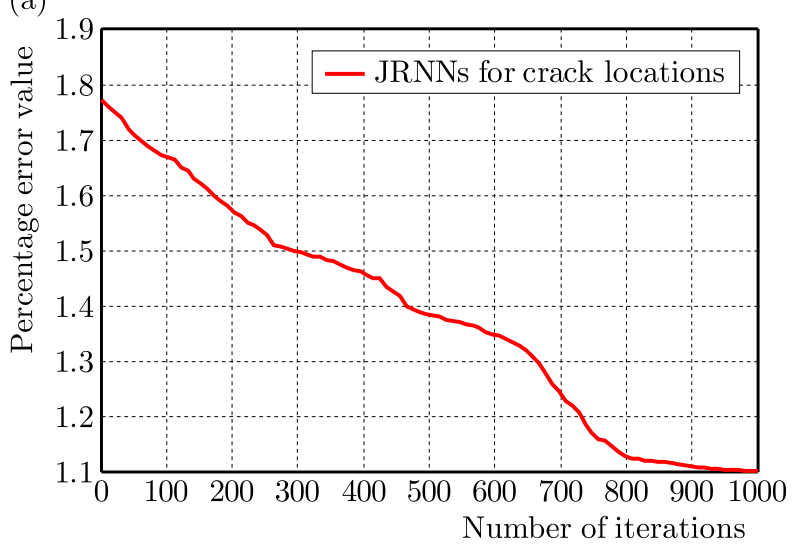

(b)

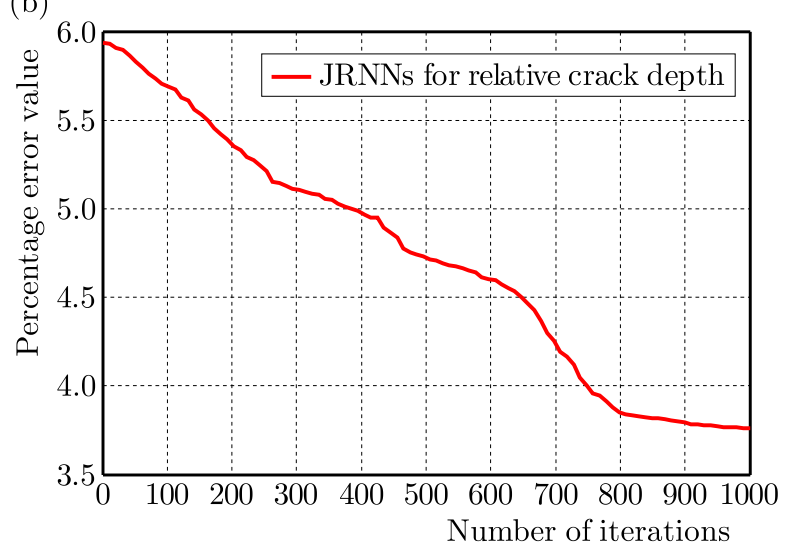

Fig. 5. Graph for iterations vs. percentage error of JRNNs for: (a) crack locations,

(b) relative crack depth

\section{Conclusions}

The response analysis along with the fault detection of a cracked simply supported beam subjected to a transiting mass is carried out in the present study. The analytical studies along with laboratory tests are considered as a forward problem and the modified JRNNs with L-M back propagation algorithm as a reverse problem. The entire studies are conducted in a supervised manner. The positions and severities of cracks are determined both by an experiment and the proposed JRNNs methods, and then compared with the numerical values. The results achieved 
from the JRNNs are quite convergent and acceptable. So, this method can be applicable to the structural health monitoring problem in the era of structural dynamics.

\section{References}

1. Bandara R.P., Chan T.H.T., Thambiratnam D.P., 2014, Structural damage detection method using frequency response functions, Structural Health Monitoring, 13, 4, 418-429

2. Bu Q.J., LAW S.S., ZhU Q,X., 2006, Innovative bridge condition assessment from dynamic response of a passing vehicle, Journal of Engineering Mechanics, ASCE, 132, 1372-1377

3. CobAn R., 2013, A context layered locally recurrent neural network for dynamic system identification, Engineering Applications of Artificial Intelligence, 26, 241-250

4. Dems K., Mróz Z., 2001, Identification of damage in beam and plate structures using parameter dependent frequency changes, Engineering Computation, 18, 1/2, 96-120

5. Ekici S., Yildirim S., Poyraz,, M., 2009, A transmission line fault locator based on Elman recurrent networks, Applied Soft Computing, 9, 341-347

6. Ettefagh M.M., Akbari H., Asadi K., Abbasi F., 2014, New structural damage-identification method using modal updating and model reduction, Journal of Mechanical Engineering Science, 229, 6, 1041-1059

7. GonzÁlez-PÉrez C., VAldÉs-GonzÁlez J., 2011, Identification of structural damage in a vehicular bridge using artificial neural networks, Structural Health Monitoring, 10, 1, 33-48

8. He W.-Y., Zhu S., 2016, Moving load-induced response of damaged beam and its application in damage localization, Journal of Vibration and Control, 22, 16, 3601-3617

9. Hu X., Balasubramaniam P., 2008, Case studies for applications of Elman recurrent neural networks, Recurrent Neural Network, 357-378, InTech Publications, Shanghai, China

10. Jena S., Parhi D.R., 2017a, Dynamic response and analysis of cracked beam subjected to transit mass, International Journal of Dynamics and Control, DOI: 10.1007/s40435-017-0361-3

11. Jena S., PARhi D.R., 2017b, Parametric study on the response of cracked structure subjected to moving mass, Journal of Vibration Engineering and Technologies, 5, 1, 11-19

12. Jena S., PARhi D.R., 2017c, Response analysis of cracked structure subjected to transit mass a parametric study, Journal of Vibroengineering, 19, 5, 3243-3254

13. Jena S., Parhi D.R., Mishra D., 2015, Comparative study on cracked beam with different types of cracks carrying moving mass, Structural Engineering and Mechanics, International Journal, 56, $5,797-811$

14. Jena S., Parhi D.R., Subbaratnam B., 2017, Parametric evaluation on the response of damaged simple supported structure under transit mass, ASME 2017 Gas Turbine India Conference

15. Kong X., Cai C.S., Kong B., 2015, Damage detection based on transmissibility of a vehicle and bridge coupled system, Journal of Engineering Mechanics, ASCE, 114, 1, 1-17

16. Krawczuk M., Ostachowicz W., 1995, Modeling and vibration analysis of a cantilever composite beam with a transverse open crack, Journal of Sound and Vibration, 183, 1, 69-89

17. Lee J.J., Lee J.W., Yi J.H., Yun B.C., Jung Y.H., 2005, Neural networks-based damage detection for bridges considering errors in baseline finite element models, Journal of Sound and Vibration, 280, 555-578

18. LEE L.-T., WU J.-Y., 2014, Identification of crack locations and depths in a multi-cracks beam by using local adaptive differential quadrature method, Journal of Process Mechanical Engineering, 229, 4, 243-255

19. Li J., LAw S.S., 2012, Damage identification of a target substructure with moving load excitation, Mechanical Systems and Signal Processing, 30, 78-90 
20. Li J., LAw S.S., HaO H., 2013, Improved damage identification in bridge structures subject to moving loads: numerical and experimental studies, International Journal of Mechanical Sciences, 74, 99-111

21. Malhi A., Gao X.R., 2004, Recurrent neural networks for long-term prediction in machine condition monitoring, IMTC 2004, Como, Italy

22. Mehrjooa M., Khaji N., Moharrami H., Bahreininejad A., 2008, Damage detection of truss bridge joints using Artificial Neural Networks, Expert Systems with Applications, 35, 3, 1122-1131

23. Mousavi M., Gandomi A.H., 2018, An input-output damage detection method using static equivalent formulation of dynamic vibration, Archives of Civil and Mechanical Engineering, 18, 2, 508-514

24. NAndakumar P., Shankar K., 2014, Multiple crack damage detection of structures using the two crack transfer matrix, Structural Health Monitoring, 13, 5, 548-561

25. Oshima Y., Yамамото K., Sugiura K., 2014, Damage assessment of a bridge based on mode shapes estimated by responses of passing vehicles, Smart Structures and Systems, 13, 5, 731-753

26. Owolabi G.M., Swamidas A.S.J., Seshadri R., 2003, Crack detection in beams using changes in frequencies and amplitudes of frequency response functions, Journal of Sound and Vibration, 265, 1-22

27. Pakrashi V., O’Connor A., Basu B., 2010, A bridge-vehicle interaction based experimental investigation of damage evolution, Structural Health Monitoring, 9, 4, 285-296

28. PARhi D.R., JenA S., 2017, Dynamic and experimental analysis on response of multiple cracked structures carrying transit mass, Journal of Risk and Reliability, 231, 1, 25-35

29. Sayyad B.F., Kumar B., 2011, Identification of crack location and crack size in a simply supported beam by measurement of natural frequencies, Journal of Vibration and Control, 18, 2, 183-190

30. Seker S., Ayaz E., TurkCAn E., 2003, Elman's recurrent neural network applications to condition monitoring in nuclear power plant and rotating machinery, Engineering Applications of Artificial Intelligence, 16, 647-656

31. Thomson T.W., 1988, Theory of Vibration with Application, Third Edition, CBS Publishers \& Distributors, New Delhi, India

32. Vosough A.R., 2015, A developed hybrid method for crack identification of beams, Smart structure and System, 16, 3, 401-414

33. Yan J.Y., Cheng L., Wu Y.Z., Yam H.L., 2007, Development in vibration-based structural damage detection technique, Mechanical Systems and Signal Processing, 21, 2198-2211

34. Yeung T.W., Smith W.J., 2005, Damage detection in bridges using neural networks for pattern recognition of vibration signatures, 27, 5, 685-698

35. Yu H., Wilamowski B.M., 2011, Levenberg-Marquardt Training, The Industrial Electronics Handbook, Intelligent Systems, 2nd Edition, 12, (1-16), CRC Press, New York, US. 